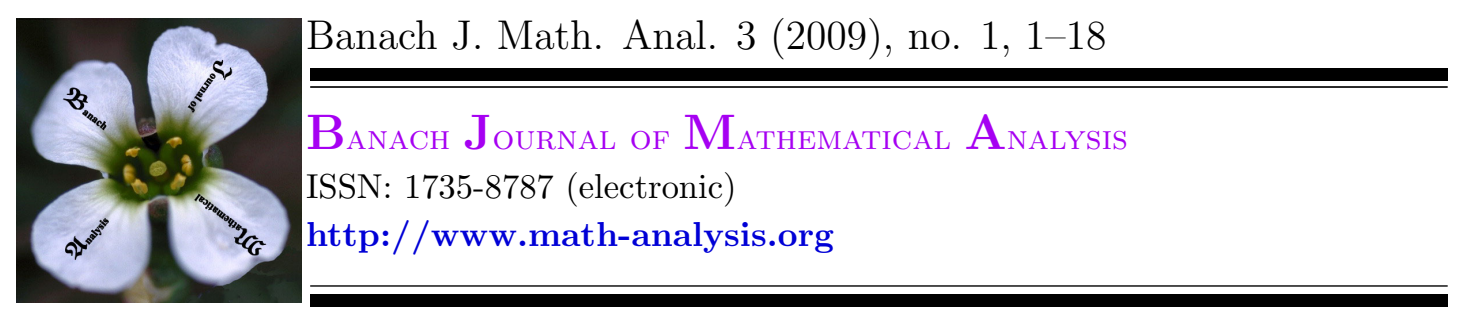

\title{
INVERTIBILITY CHARACTERIZATION OF WIENER-HOPF PLUS HANKEL OPERATORS VIA ODD ASYMMETRIC FACTORIZATIONS
}

\author{
G. BOGVERADZE ${ }^{1}$ AND L.P. CASTRO ${ }^{2 *}$ \\ Communicated by H. Dedania
}

\begin{abstract}
The invertibility of Wiener-Hopf plus Hankel operators with essentially bounded Fourier symbols is characterized via certain factorization properties of the Fourier symbols. In addition, a Fredholm criterion for these operators is also obtained and the dimensions of the kernel and cokernel are described.
\end{abstract}

\section{INTRODUCTION}

The main goal of the present work is to obtain invertibility and Fredholm criteria for Wiener-Hopf plus Hankel operators acting between $L^{2}$ Lebesgue spaces on the half-line. To be more precise, the main operators in study have therefore the form:

$$
W H_{\varphi}:=W_{\varphi}+H_{\varphi}=r_{+} \mathcal{F}^{-1} \varphi \mathcal{F}(I+J): L_{+}^{2}(\mathbb{R}) \rightarrow L^{2}\left(\mathbb{R}_{+}\right)
$$

where

$$
\begin{aligned}
W_{\varphi} & :=r_{+} \mathcal{F}^{-1} \varphi \mathcal{F}: L_{+}^{2}(\mathbb{R}) \rightarrow L^{2}\left(\mathbb{R}_{+}\right), \\
H_{\varphi} & :=r_{+} \mathcal{F}^{-1} \varphi \mathcal{F} J: L_{+}^{2}(\mathbb{R}) \rightarrow L^{2}\left(\mathbb{R}_{+}\right) .
\end{aligned}
$$

Here $L^{2}(\mathbb{R})$ and $L^{2}\left(\mathbb{R}_{+}\right)$denote the Banach spaces of complex-valued Lebesgue measurable functions $\varphi$, for which $|\varphi|^{2}$ is integrable on $\mathbb{R}$ and $\mathbb{R}_{+}$, respectively. In

Date: Received: 14 February 2008; Accepted: 18 June 2008.

* Corresponding author.

2000 Mathematics Subject Classification. Primary 47B35; Secondary 47A68, 47A53.

Key words and phrases. Wiener-Hopf operator, Hankel operator, invertibility, Fredholm property, odd asymmetric factorization. 
addition, $L_{+}^{2}(\mathbb{R})$ denotes the subspace of $L^{2}(\mathbb{R})$ formed by all functions supported in the closure of $\mathbb{R}_{+}=(0,+\infty), r_{+}$is the restriction operator from $L^{2}(\mathbb{R})$ into $L^{2}\left(\mathbb{R}_{+}\right), \mathcal{F}$ denotes the Fourier transformation, $J$ is the reflection operator given by the rule $J \varphi(x)=\widetilde{\varphi}(x)=\varphi(-x), x \in \mathbb{R}$ and $\varphi \in L^{\infty}(\mathbb{R})$ is the so-called Fourier symbol.

We would like to point out that in recent times algebraic combinations of Wiener-Hopf and Hankel operators have been receiving an increased attention in view of their invertibility and Fredholm properties. Part of this interest comes from certain applications where such combinations of operators arise. Examples of recent results within this context can be found e.g. in [1, 7, 8, 9, 10]. Some of these works propose certain asymmetric factorization concepts which are helpful to look for in view of the invertibility properties of corresponding operators with symmetries. In coherence to these developments, in the present paper we propose an odd asymmetric factorization concept which will be crucial to find an invertibility and Fredholm characterization for Wiener-Hopf plus Hankel operators with essentially bounded Fourier symbols.

It should be mentioned that several partial results about Fredholm characteristics and invertibility of Wiener-Hopf plus Hankel operators are presently known. Namely, for such operators with continuous or piecewise continuous Fourier symbols corresponding Fredholm characterizations are known for some time now. In addition, for Fourier symbols in the algebra of semi-almost periodic functions, conditions which ensure the Fredholm property (or the lateral invertibility) of the corresponding Wiener-Hopf plus Hankel operators were recently described in $[4,5,11,13]$ (e.g., upon certain mean values of the representatives at infinity of their Fourier symbols). Also for unitary and sectorial Fourier symbols, an invertibility criterion of Wiener-Hopf plus Hankel operators can be found in [3].

Still concerned with the context of the present work, it should be also mentioned that a factorization theory for the class of Toeplitz plus Hankel operators was proposed in [2]. Such factorization theory leads to invertibility and Fredholm criteria for Toeplitz plus Hankel operators with the same symbols. Thus, by using some known operator identities, it is possible to derive from [2] a corresponding criteria for Wiener-Hopf minus Hankel operators. However, the situation of Wiener-Hopf plus Hankel operators considered in the present paper is different, and cannot simply be taken from the results of [2]. The reason for this is structural since e.g. we can exhibit for the same Fourier symbols Wiener-Hopf plus Hankel operators which are Fredholm and corresponding Wiener-Hopf minus Hankel operators which are not Fredholm (and vice-versa); the same can be seen for the invertibility property, etc. In addition, we would also like to point out that the work [12] is engaged with a factorization theory exactly for Wiener-Hopf plus Hankel operators but with almost periodic Fourier symbols.

The paper is organized in the following way. In the next section some auxiliary operators and identities are recalled. The new concepts of weak odd asymmetric factorization and (strong) odd asymmetric factorization are introduced in Section 3, as well as the main statement of the present work about the invertibility of Wiener-Hopf plus Hankel operators with essentially bounded Fourier symbols 
(Theorem 3.4). Section 4 is devoted to the analysis of some auxiliary results which will be used in Section 5 where the proof of Theorem 3.4 is performed. Finally, a Fredholm criterion for $W_{\varphi}$ is obtained in Section 6 together with a description of the dimensions of its kernel and cokernel.

\section{BASIC NOTATION AND IDENTITIES}

Taking profit of the notations (1.1)-(1.2), we start by recalling a basic formula from the theory of Wiener-Hopf-Hankel operators which will be used in the next sections:

$$
W H_{\varphi \psi}=W H_{\varphi} \ell_{0} W H_{\psi}+H_{\varphi} \ell_{0} W H_{\widetilde{\psi}-\psi}
$$

where $\ell_{0}: L^{2}\left(\mathbb{R}_{+}\right) \rightarrow L_{+}^{2}(\mathbb{R})$ denotes the zero extension operator.

We will now present some additional operators (and notation) which will be useful in what follows. We will use the operator $W_{\varphi}^{0}$ of convolution with symbol $\varphi$ (acting between $L^{2}(\mathbb{R})$ spaces), i.e.,

$$
W_{\varphi}^{0}=\mathcal{F}^{-1} \varphi \mathcal{F}: L^{2}(\mathbb{R}) \rightarrow L^{2}(\mathbb{R})
$$

The Cauchy singular integral operator $S_{\mathbb{R}}$ on $L^{2}(\mathbb{R})$ is defined by:

$$
\left(S_{\mathbb{R}} f\right)(x)=\frac{1}{\pi i} \int_{\mathbb{R}} \frac{f(s)}{s-x} d s, \quad x \in \mathbb{R},
$$

where the integral is understood in the principal value sense. The Riesz projection is given by the following formula

$$
P_{\mathbb{R}}=\frac{1}{2}\left(I+S_{\mathbb{R}}\right)
$$

and we will also be dealing with the complementary projection $Q_{\mathbb{R}}=I-P_{\mathbb{R}}$. The analogues of these operators for the unit circle $\Gamma$ will also be used. Namely, the Cauchy singular integral operator on the unit circle $S_{\Gamma}$, the Riesz projection for the unit circle $P_{\Gamma}$ and its complementary projection $Q_{\Gamma}$ (which are defined in an analogous way).

The Toeplitz operator is given by the following formula:

$$
T_{\phi}=P_{\Gamma} L(\phi) P_{\Gamma}: H_{+}^{2}(\Gamma) \rightarrow H_{+}^{2}(\Gamma),
$$

where $L(\phi)$ is simply the multiplication operator defined on $L^{2}(\Gamma)$. The notation $H_{ \pm}^{p}(X)(1 \leq p \leq \infty)$ stands for the usual Hardy spaces on $X$. The Hankel operator for the unit circle is given by:

$$
H_{\phi}=P_{\Gamma} L(\phi) J_{\Gamma} P_{\Gamma}: H_{+}^{2}(\Gamma) \rightarrow H_{+}^{2}(\Gamma),
$$

where $J_{\Gamma}: L^{2}(\Gamma) \rightarrow L^{2}(\Gamma)$ is a Carleman shift operator, which acts by the rule:

$$
\left(J_{\Gamma} f\right)(t)=\frac{1}{t} f\left(\frac{1}{t}\right) .
$$

Hence, the Toeplitz minus Hankel operator is defined by:

$$
T_{\phi}-H_{\phi}=P_{\Gamma} L(\phi)\left(I-J_{\Gamma}\right) P_{\Gamma}: H_{+}^{2}(\Gamma) \rightarrow H_{+}^{2}(\Gamma) .
$$

In case a function $f$ is defined on the unit circle, the previous notation $\tilde{f}$ will have now the meaning $\widetilde{f}(t):=f\left(t^{-1}\right), t \in \Gamma$. As usual, on the unit circle $\Gamma$, we say 
that a function $f$ is even if $\tilde{f}=f$ and $f$ is said to be an odd function if $\tilde{f}=-f$. These definitions justify the use of the same tilde notation in both settings $\Gamma$ and $\mathbb{R}$.

\section{ODD FACTORIZATIONS ON THE REAL LINE AND MAIN INVERTIBILITY RESULT}

We start this section by introducing a definition that will be later on completed by a corresponding stronger version which will have a central role in our invertibility and Fredholm criteria for the Wiener-Hopf plus Hankel operators. Thus, at the end of the present section it will be already possible to state the main invertibility criterion for $\mathrm{WH}_{\varphi}$ (cf. (1.1)).

Definition 3.1. A function $\varphi \in \mathcal{G} L^{\infty}(\mathbb{R})$ is said to admit a weak odd asymmetric factorization in $L^{2}(\mathbb{R})$ if it admits a representation

$$
\varphi(x)=\varphi_{-}(x)\left(\frac{x-i}{x+i}\right)^{m} \varphi_{o}(x), \quad x \in \mathbb{R},
$$

such that $m \in \mathbb{Z}$, and

$$
\begin{aligned}
& \text { (i) } \quad \frac{x}{(x-i)^{2}} \varphi_{-} \in H_{-}^{2}(\mathbb{R}), \quad \frac{1}{(x-i)^{2}} \varphi_{-}^{-1} \in H_{-}^{2}(\mathbb{R}), \\
& \text { (ii) } \quad \frac{1}{x^{2}+1} \varphi_{o} \in L_{\text {odd }}^{2}(\mathbb{R}), \quad \frac{|x|}{x^{2}+1} \varphi_{o}^{-1} \in L_{\text {odd }}^{2}(\mathbb{R}) \text {. }
\end{aligned}
$$

Here $L_{\text {odd }}^{2}(X)$ stands for the class of odd functions from the space $L^{2}(X)$. The integer $m$ is called the index of the weak odd asymmetric factorization (3.1) in $L^{2}(\mathbb{R})$.

Let us note that we have the uniqueness (up to a constant) of such type of factorizations. This last property is given in exact terms in the next theorem.

Theorem 3.2. Assume that $\varphi \in \mathcal{G} L^{\infty}(\mathbb{R})$ admits two weak odd asymmetric factorizations in $L^{2}(\mathbb{R})$ :

$$
\varphi(x)=\varphi_{-}^{(1)}(x)\left(\frac{x-i}{x+i}\right)^{\kappa_{1}} \varphi_{o}^{(1)}(x)=\varphi_{-}^{(2)}(x)\left(\frac{x-i}{x+i}\right)^{\kappa_{2}} \varphi_{o}^{(2)}(x), \quad x \in \mathbb{R} .
$$

Then, we necessarily have $\kappa_{1}=\kappa_{2}, \varphi_{-}^{(1)}=C \varphi_{-}^{(2)}$ and $\varphi_{o}=C^{-1} \varphi_{o}^{(2)}$, for some constant $C \in \mathbb{C} \backslash\{0\}$.

Proof. Let $\varphi$ admit two weak odd asymmetric factorizations:

$$
\varphi(x)=\varphi_{-}^{(1)}(x)\left(\frac{x-i}{x+i}\right)^{\kappa_{1}} \varphi_{o}^{(1)}(x)=\varphi_{-}^{(2)}(x)\left(\frac{x-i}{x+i}\right)^{\kappa_{2}} \varphi_{o}^{(2)}(x), x \in \mathbb{R}
$$

(where $\varphi_{-}^{(1)}, \varphi_{-}^{(2)}$ and $\varphi_{o}^{(1)}, \varphi_{o}^{(2)}$ have the corresponding properties of (i) and (ii) in Definition 3.1). From (3.2) we immediately have that

$$
\varphi_{-}^{(1)}(x)\left(\varphi_{-}^{(2)}(x)\right)^{-1}\left(\frac{x-i}{x+i}\right)^{\kappa_{1}-\kappa_{2}}=\varphi_{o}^{(2)}(x)\left(\varphi_{o}^{(1)}(x)\right)^{-1}, \quad x \in \mathbb{R} .
$$


We can assume without lost of generality that $\kappa:=\kappa_{1}-\kappa_{2} \leq 0$, since otherwise we would consider

$$
\varphi_{-}^{(2)}(x)\left(\varphi_{-}^{(1)}(x)\right)^{-1}\left(\frac{x-i}{x+i}\right)^{\kappa_{2}-\kappa_{1}}=\varphi_{o}^{(1)}(x)\left(\varphi_{o}^{(2)}(x)\right)^{-1}, \quad x \in \mathbb{R}
$$

instead of (3.3) (and from this last identity we are able to take the same conclusion and therefore proceed with the proof in a similar way).

Let us now consider the following auxiliary function:

$$
\psi(x):=\frac{x}{(x-i)^{4}} \varphi_{-}^{(1)}(x)\left(\varphi_{-}^{(2)}(x)\right)^{-1} \in H_{-}^{1}(\mathbb{R}) .
$$

A direct computation yields that

$$
\widetilde{\psi}(x):=\frac{-x}{(x+i)^{4}}{\widetilde{\varphi_{-}}}^{(1)}(x)\left({\widetilde{\varphi_{-}}}^{(2)}(x)\right)^{-1} \in H_{+}^{1}(\mathbb{R}) .
$$

The right hand side of (3.3) is an even function (since it is a product of two odd functions). Hence, from (3.3), we immediately obtain that

$$
\varphi_{-}^{(1)}(x)\left(\varphi_{-}^{(2)}(x)\right)^{-1}\left(\frac{x-i}{x+i}\right)^{2 \kappa}=\widetilde{\varphi}_{-}^{(1)}(x)\left(\widetilde{\varphi}_{-}^{(2)}(x)\right)^{-1} .
$$

This identity together with (3.4) and (3.5), lead to the conclusion that

$$
\psi(x)\left(\frac{x-i}{x+i}\right)^{2 \kappa+4}=-\widetilde{\psi}(x) .
$$

Due to the inclusions in (3.4) and (3.5), if $2 \kappa+4 \leq 0$ then from (3.6) we immediately obtain that $\psi=0$ is identically zero and hence we would have a contradiction. This means that it only remains the possibilities of $\kappa=-1$ and $\kappa=0$.

Let us analyze the case where $\kappa=-1$. In the present case, (3.6) is reduced to the form

$$
(x-i)^{2} \psi(x)=-(x+i)^{2} \widetilde{\psi}(x) .
$$

Hence, using (3.4)-(3.5), we have a contradiction which shows that $\kappa$ cannot be equal to -1 .

Thus, the only possibility which is left for $\kappa$ is to be equal to zero. Therefore, in such a case, $\kappa_{1}=\kappa_{2}$. In this case we will have that

$$
\varphi_{-}^{(1)}(x)\left(\varphi_{-}^{(2)}(x)\right)^{-1}={\widetilde{\varphi_{-}}}^{(1)}(x)\left(\widetilde{\varphi}_{-}^{(2)}(x)\right)^{-1} .
$$

Consequently, $\varphi_{-}^{(1)}(x)\left(\varphi_{-}^{(2)}(x)\right)^{-1}=C$ for a constant $C \in \mathbb{C} \backslash\{0\}$. Thus $\varphi_{-}^{(1)}=$ $C \varphi_{-}^{(2)}$ and $\varphi_{o}^{(1)}=C^{-1} \varphi_{o}^{(2)}$.

The following definition may be viewed as a strong version of the previous introduced weak factorization and will play a crucial role in the main theorem below. 
Definition 3.3. A function $\varphi \in \mathcal{G} L^{\infty}(\mathbb{R})$ is said to admit an odd asymmetric factorization in $L^{2}(\mathbb{R})$ if it admits a representation

$$
\varphi(x)=\varphi_{-}(x)\left(\frac{x-i}{x+i}\right)^{m} \varphi_{o}(x), \quad x \in \mathbb{R},
$$

such that $m \in \mathbb{Z}$, and

$$
\begin{array}{ll}
\frac{x}{(x-i)^{2}} \varphi_{-} \in H_{-}^{2}(\mathbb{R}), & \frac{1}{(x-i)^{2}} \varphi_{-}^{-1} \in H_{-}^{2}(\mathbb{R}), \\
\frac{1}{\left(x^{2}+1\right)} \varphi_{o} \in L_{\text {odd }}^{2}(\mathbb{R}), & \frac{|x|}{\left(x^{2}+1\right)} \varphi_{o}^{-1} \in L_{\text {odd }}^{2}(\mathbb{R}),
\end{array}
$$

(iii) the linear operator $\mathscr{S}:=W_{\varphi_{o}^{-1}}^{0}(I-J) \ell_{0} W_{\varphi_{-}^{-1}}: L^{2}(\mathbb{R}) \rightarrow L_{\text {even }}^{2}(\mathbb{R})$ is bounded.

The integer $m$ is called the index of the odd asymmetric factorization (3.7) in $L^{2}(\mathbb{R})$.

We are now in a position to state the main result about the invertibility of our Wiener-Hopf plus Hankel operators with $L^{\infty}$ symbols.

Theorem 3.4. Let $\varphi \in \mathcal{G} L^{\infty}(\mathbb{R})$. The operator $W H_{\varphi}$ is invertible if and only if $\varphi$ admits an odd asymmetric factorization in $L^{2}(\mathbb{R})$ with index $m=0$.

The proof of this theorem will be given in Section 5 .

\section{OdD FACTORIZATIONS ON THE UNIT CIRCLE}

In the present section we will introduce some auxiliary notions which will be useful to work out some conclusions in the unit circle setting.

Definition 4.1. A function $\phi \in \mathcal{G} L^{\infty}(\Gamma)$ is said to admit a weak odd asymmetric factorization in $L^{2}(\Gamma)$ if it admits a representation

$$
\phi(t)=\phi_{-}(t) t^{k} \phi_{o}(t), \quad t \in \Gamma
$$

such that $k \in \mathbb{Z}$ and

$$
\begin{array}{ll}
\text { (i) } & \left(1+t^{-1}\right) \phi_{-} \in H_{-}^{2}(\Gamma), \quad\left(1-t^{-1}\right) \phi_{-}^{-1} \in H_{-}^{2}(\Gamma), \\
\text { (ii }) & |1-t| \phi_{o} \in L_{\text {odd }}^{2}(\Gamma), \quad|1+t| \phi_{o}^{-1} \in L_{\text {odd }}^{2}(\Gamma) .
\end{array}
$$

The integer $k$ is called the index of an asymmetric factorization in $L^{2}(\Gamma)$.

Now we will present a theorem about the uniqueness of a weak odd asymmetric factorization in $L^{2}(\Gamma)$.

Theorem 4.2. Assume that $\phi$ admits two weak odd asymmetric factorizations in $L^{2}(\Gamma)$ :

$$
\phi(t)=\phi_{-}^{(1)}(t) t^{k_{1}} \phi_{o}^{(1)}(t)=\phi_{-}^{(2)}(t) t^{k_{2}} \phi_{o}^{(2)}(t), \quad t \in \Gamma .
$$

Then $k_{1}=k_{2}, \phi_{-}^{(1)}=C \phi_{-}^{(2)}, \phi_{o}^{(1)}=C^{-1} \phi_{o}^{(2)}$ with $C \in \mathbb{C} \backslash\{0\}$. 
Proof. Without lost of generality, we can assume that $m=k_{1}-k_{2} \leq 0$. From (4.1) we have that

$$
\left(\phi_{-}^{(2)}\right)^{-1} \phi_{-}^{(1)} t^{m}=\phi_{o}^{(2)}\left(\phi_{o}^{(1)}\right)^{-1} .
$$

Take $\psi=\left(1-t^{-2}\right)\left(\phi_{-}^{(2)}\right)^{-1} \phi_{-}^{(1)}$. Obviously, we have that $\psi \in H_{-}^{1}(\Gamma)$. Formula (4.2) leads to

$$
\left(1-t^{-2}\right)^{-1} \psi(t) t^{m}=\phi_{o}^{(2)}(t)\left(\phi_{o}^{(1)}(t)\right)^{-1},
$$

where the right hand side is an even function (since it is a product of two odd functions). Therefore,

$$
\left(1-t^{-2}\right)^{-1} \psi(t) t^{m}=\left(1-t^{2}\right)^{-1} \psi\left(t^{-1}\right) t^{-m},
$$

and from here we have:

$$
\psi(t) t^{2 m+2}=-\psi\left(t^{-1}\right) .
$$

If we assume that $m \leq-1$ we would obtain that $\psi=0$ (by observing the Fourier coefficients of $\psi$ ), which is a contradiction. Hence $m=0$. In this case we have that $\psi(t)=C\left(1-t^{-2}\right)$ with $C \neq 0$. Finally, from here we have: $k_{1}=k_{2}, \phi_{-}^{(1)}=C \phi_{-}^{(2)}$, and $\phi_{o}^{(1)}=C^{-1} \phi_{o}^{(2)}$.

Consider the following complementary projections:

$$
P_{J_{\Gamma}}:=\frac{I+J_{\Gamma}}{2}: L^{2}(\Gamma) \rightarrow L^{2}(\Gamma), \quad Q_{J_{\Gamma}}:=I-P_{J_{\Gamma}} .
$$

These projections decompose $L^{2}(\Gamma)$ into the direct sum: $L^{2}(\Gamma)=\operatorname{Im} P_{J_{\Gamma}} \oplus \operatorname{Im} Q_{J_{\Gamma}}$.

Let $\mathfrak{R}$ stand for the linear space of all trigonometric polynomials. A natural strong version of the Definition 4.1 is given next.

Definition 4.3. A function $\phi \in \mathcal{G} L^{\infty}(\Gamma)$ is said to admit an odd asymmetric factorization in $L^{2}(\Gamma)$ if it admits a representation

$$
\phi(t)=\phi_{-}(t) t^{k} \phi_{o}(t), \quad t \in \Gamma
$$

such that $k \in \mathbb{Z}$ and

$$
\begin{aligned}
& \left(1+t^{-1}\right) \phi_{-} \in H_{-}^{2}(\Gamma), \quad\left(1-t^{-1}\right) \phi_{-}^{-1} \in H_{-}^{2}(\Gamma), \\
& |1-t| \phi_{o} \in L_{\text {odd }}^{2}(\Gamma), \quad|1+t| \phi_{o}^{-1} \in L_{\text {odd }}^{2}(\Gamma), \\
& \text { the linear operator } \mathscr{E}=L\left(\phi_{o}^{-1}\right)\left(I+J_{\Gamma}\right) P_{\Gamma} L\left(\phi_{-}^{-1}\right) \text { acting from } \\
& X_{1}:=\left\{\left(1-t^{-1}\right) f(t): f \in \mathfrak{R}\right\} \text { into } X_{2}:=\left\{\left(1+t^{-1}\right) \phi_{o}^{-1}(t) f(t): f \in \mathfrak{R},\right. \\
& \left.f(t)=f\left(t^{-1}\right)\right\} \text { extends to a linear bounded operator } \widetilde{\mathscr{E}} \text { acting from } \\
& L^{2}(\Gamma) \text { into } \operatorname{Im} Q_{J_{\Gamma}} .
\end{aligned}
$$

Accordingly as before, also in here $k$ is called the index of the odd asymmetric factorization in $L^{2}(\Gamma)$.

Taking into account that $X_{1}$ is a dense subset of $L^{2}(\Gamma)$, an equivalent formulation of the condition (iii) is evidently the following:

$\left(i i i^{*}\right) \quad$ the operator $\mathscr{E}$ is a bounded operator on $L^{2}(\Gamma)$. 
We observe that Definition 4.3 is related with Definition 3.3 in the sense that a function $\phi: \Gamma \rightarrow \mathbb{C}$ admits an odd asymmetric factorization in $L^{2}(\Gamma)$ if and only if the function $\varphi(x):=\phi\left(\frac{x-i}{x+i}\right), x \in \mathbb{R}$, admits an odd asymmetric factorization in $L^{2}(\mathbb{R})$. To present such a relation in an explicit way, let us consider the useful operator $B_{0}$ given by

$$
\left(B_{0} \varphi\right)(t)=\varphi\left(i \frac{1+t}{1-t}\right), \quad t \in \Gamma .
$$

Obviously $B_{0}: L^{\infty}(\mathbb{R}) \rightarrow L^{\infty}(\Gamma)$ is an isometrical isomorphism, the inverse of which is given by the following formula:

$$
\left(B_{0}^{-1} \phi\right)(x)=\phi\left(\frac{x-i}{x+i}\right), \quad x \in \mathbb{R} .
$$

In addition, the operator $B$ given by

$$
(B \varphi)(x)=\frac{\sqrt{2}}{x+i} \varphi\left(\frac{x-i}{x+i}\right), \quad x \in \mathbb{R},
$$

is an isometrical isomorphism of $L^{2}(\Gamma)$ onto $L^{2}(\mathbb{R})$, of $H_{+}^{2}(\Gamma)$ onto $H_{+}^{2}(\mathbb{R})$, and of $t^{-1} H_{-}^{2}(\Gamma)$ onto $H_{-}^{2}(\mathbb{R})$. For the inverse of $B$, we have:

$$
\left(B^{-1} \phi\right)(t)=\frac{i \sqrt{2}}{1-t} \phi\left(i \frac{1+t}{1-t}\right), \quad t \in \Gamma .
$$

By using the "convolution" with $B$ operators, it is obtained the formula:

$$
B L(\phi) B^{-1}=\left(B_{0}^{-1} \phi\right) I
$$

A straightforward computation shows that

$$
S_{\mathbb{R}}=B S_{\Gamma} B^{-1}, \quad P_{\mathbb{R}}=B P_{\Gamma} B^{-1}, \quad Q_{\mathbb{R}}=B Q_{\Gamma} B^{-1}
$$

The following formula is also of interest:

$$
\mathcal{F}^{-1} P_{\mathbb{R}} \mathcal{F}=\ell_{0} r_{+}: L^{2}(\mathbb{R}) \rightarrow L_{+}^{2}(\mathbb{R}) .
$$

Let $\mathcal{L}(X, Y)$ denote the Banach space of all bounded linear operators acting between Banach spaces $X$ and $Y$. In the case of $X=Y$, we will abbreviate $\mathcal{L}(X, X)$ by $\mathcal{L}(X)$.

In what follows we will also use the notion of equivalent operators in the following way. Let $T \in \mathcal{L}\left(Z_{1}, Y_{1}\right)$ and $S \in \mathcal{L}\left(Z_{2}, Y_{2}\right)$ (for Banach spaces $Z_{1}, Z_{2}, Y_{1}$ and $\left.Y_{2}\right)$. It is said that $T$ and $S$ are equivalent operators, if there exist bounded invertible operators $E \in \mathcal{L}\left(Y_{2}, Y_{1}\right)$ and $F \in \mathcal{L}\left(Z_{1}, Z_{2}\right)$, such that $T=E S F$. In addition, if we have an equality $T=E S E^{-1}$ we say that $T$ and $S$ are unitarily equivalent operators.

Proposition 4.4. A function $\phi \in \mathcal{G} L^{\infty}(\Gamma)$ admits an odd asymmetric factorization in $L^{2}(\Gamma)$ with index $k$ if and only if $\varphi:=\left(B_{0}^{-1} \phi\right) \in \mathcal{G} L^{\infty}(\mathbb{R})$ admits an odd asymmetric factorization in $L^{2}(\mathbb{R})$ with index $k$. 
Proof. Let us assume that $\phi$ admits an odd asymmetric factorization in $L^{2}(\Gamma)$ with index $k$. Hence, we can write (cf. (4.3)):

$$
\phi(t)=\phi_{-}(t) t^{k} \phi_{o}(t), \quad t \in \Gamma
$$

with the properties $(i)-($ iii $)$ on the factors stated in Definition 4.3. Performing the $B_{0}^{-1}$ transformation in both sides of the equality (4.7), we obtain:

$$
\left(B_{0}^{-1} \phi\right)(x)=\left(B_{0}^{-1} \phi_{-}\right)(x)\left(B_{0}^{-1} d\right)(x)\left(B_{0}^{-1} \phi_{o}\right)(x),
$$

where $d$ denotes the function $d(t):=t^{k}$. Now, if defining

$$
\begin{gathered}
\varphi(x):=\left(B_{0}^{-1} \phi\right)(x)=\phi\left(\frac{x-i}{x+i}\right), \\
\varphi_{-}(x):=\left(B_{0}^{-1} \phi_{-}\right)(x)=\phi_{-}\left(\frac{x-i}{x+i}\right), \\
\varphi_{o}(x):=\left(B_{0}^{-1} \phi_{o}\right)(x)=\phi_{o}\left(\frac{x-i}{x+i}\right),
\end{gathered}
$$

it follows

$$
\varphi(x)=\varphi_{-}(x)\left(\frac{x-i}{x+i}\right)^{k} \varphi_{o}(x) .
$$

I.e., formula (3.7) with $m$ taken to be equal to $k$. Thus, we are left to show that the corresponding conditions $(i)-($ iii $)$ on the factors used in the factorizations of definitions 4.3 and 3.3 are equivalent.

We have that

$$
\left(1+t^{-1}\right) \phi_{-} \in H_{-}^{2}(\Gamma)
$$

if and only if

$$
\frac{\sqrt{2}}{x-i}\left(1+\frac{x+i}{x-i}\right) \varphi_{-} \in H_{-}^{2}(\mathbb{R}) .
$$

Indeed, let $\left(1+t^{-1}\right) \phi_{-} \in H_{-}^{2}(\Gamma)$, then $\left[B_{0}^{-1}\left(1+t^{-1}\right) \phi_{-}\right](x) \in(x-i) H_{-}^{2}(\mathbb{R})$ (cf., e.g., [6, page 108 and in particular formula (6.3)]). That means

$$
2 \sqrt{2} \frac{x}{(x-i)^{2}} \varphi_{-}(x)=\frac{\sqrt{2}}{x-i}\left(1+\frac{x+i}{x-i}\right) \varphi_{-}(x) \in H_{-}^{2}(\mathbb{R}),
$$

and therefore we have the equivalence of the first propositions of conditions $(i)$.

To prove the equivalence of the first proposition of $(i i)$-conditions we need to "compensate" the space with a particular even weight. Letting $|1-t| \phi_{o} \in L_{\text {odd }}^{2}(\Gamma)$, then

$$
B_{0}^{-1}\left(|1-t| \phi_{o}\right) \in B_{0}^{-1}\left(L_{\text {odd }}^{2}(\Gamma)\right) .
$$

Thus, to obtain from the last inclusion a new one where we will be dealing with the space $L_{\text {odd }}^{2}(\mathbb{R})$ we just need to use in (4.8) the multiplication by the weight function $\frac{1}{\sqrt{x^{2}+1}}$ and therefore reach to

$$
\frac{1}{\sqrt{x^{2}+1}}\left(B_{0}^{-1}\left(|1-t| \phi_{o}\right)\right)(x) \in L_{\text {odd }}^{2}(\mathbb{R}) \text {. }
$$


Consequently, we have:

$$
\frac{2}{x^{2}+1} \varphi_{o}(x) \in L_{\text {odd }}^{2}(\mathbb{R}) .
$$

Analogous arguments will give corresponding equivalences for the second inclusions in conditions $(i)$ and $(i i)$.

We will prove now the equivalence of conditions (iii). As far as the condition ( iii) of Definition 4.3 can be written in the form of the condition $\left(i i i^{*}\right)$ cited after Definition 4.3 , we will show that $\mathscr{E}$ is a bounded operator if and only if $\mathscr{S}$ is a bounded operator. Consider the following operator:

$$
\mathcal{F}^{-1} B \mathscr{E} B^{-1} \mathcal{F} \text {. }
$$

This operator is equivalent to $\mathscr{E}$ simply because it is obtained from $\mathscr{E}$ by multiplying from the left and from the right by invertible operators. Moreover, from (4.9) we have:

$$
\begin{aligned}
\mathcal{F}^{-1} B \mathscr{E} B^{-1} \mathcal{F}= & \mathcal{F}^{-1} B L\left(\phi_{o}^{-1}\right)\left(I+J_{\Gamma}\right) P_{\Gamma} L\left(\phi_{-}^{-1}\right) B^{-1} \mathcal{F} \\
= & \mathcal{F}^{-1} B L\left(\phi_{o}^{-1}\right) \underbrace{B^{-1} \mathcal{F} \mathcal{F}^{-1} B}_{I}\left(I+J_{\Gamma}\right) \underbrace{B^{-1} \mathcal{F} \mathcal{F}^{-1} B}_{I} P_{\Gamma} \\
& \underbrace{B^{-1} \mathcal{F} \mathcal{F}^{-1} B}_{I} L\left(\phi_{-}^{-1}\right) B^{-1} \mathcal{F} \\
= & \mathcal{F}^{-1} \varphi_{o}^{-1} \mathcal{F}(I-J) \ell_{0} r_{+} \mathcal{F}^{-1} \varphi_{-}^{-1} \mathcal{F} \\
= & W_{\varphi_{o}^{-1}}^{0}(I-J) \ell_{0} W_{\varphi_{-}^{-1}}=\mathscr{S}
\end{aligned}
$$

where we have used formulas (4.4), (4.5) and (4.6). Finally this means that $\mathscr{E}$ and $\mathscr{S}$ are unitarily equivalent operators.

From the above reasoning it is clear that we can proceed in a "reverse" direction, i.e., starting from a factorization for the function $\varphi$ and obtain a corresponding factorization to the function $\phi$, which completes the proof.

\section{ProOF OF THE INVERTIBILITY CRITERION}

To prove the main invertibility result of the present work (i.e., Theorem 3.4) we need first to prepare some auxiliary material.

5.1. Auxiliary notions, operators, and results. We will relate Toeplitz minus Hankel operators with the following operators:

$$
\begin{aligned}
& \overrightarrow{T_{\phi}}=P_{\Gamma} L(\phi) Q_{J_{\Gamma}}: \operatorname{Im} Q_{J_{\Gamma}} \rightarrow H_{+}^{2}(\Gamma) \\
& \overleftarrow{T_{\psi}}=Q_{J_{\Gamma}} L(\psi) P_{\Gamma}: H_{+}^{2}(\Gamma) \rightarrow \operatorname{Im} Q_{J_{\Gamma}}
\end{aligned}
$$

where $\psi(t)=\phi^{-1}\left(-t^{-1}\right)$. It is readily seen that $2 \overrightarrow{T_{\phi}}=\left.\left(T_{\phi}-H_{\phi}\right)\right|_{\operatorname{Im} Q_{J_{\Gamma}}}$.

The following well-known lemma is of interest and will be used to prove Proposition 5.3.

Lemma 5.1. Let $Z_{1}$ and $Z_{2}$ be linear spaces, $A: Z_{1} \rightarrow Z_{2}$ be a linear and invertible operator, $P_{1}: Z_{1} \rightarrow Z_{1}$ and $P_{2}: Z_{2} \rightarrow Z_{2}$ be linear projections, and $Q_{1}=I-P_{1}$ and $Q_{2}=I-P_{2}$. Then $P_{2} A P_{1}: \operatorname{Im} P_{1} \rightarrow \operatorname{Im} P_{2}$ is invertible if and only if $Q_{1} A^{-1} Q_{2}: \operatorname{Im} Q_{2} \rightarrow \operatorname{Im} Q_{1}$ is invertible. 
The proof of Lemma 5.1 can be found e.g. in [14].

We will now present two basic propositions which anyway will turn clear the reason why to consider the above introduced operators $\vec{T}_{\phi}$ and $\overleftarrow{T_{\psi}}$.

Proposition 5.2. Let $\phi \in \mathcal{G} L^{\infty}(\Gamma)$. The operator $\vec{T}_{\phi} \in \mathcal{L}\left(\operatorname{Im} Q_{J_{\Gamma}}, H_{+}^{2}(\Gamma)\right)$ (defined in (5.1)) is equivalent to the Toeplitz minus Hankel operator $T_{\phi}-H_{\phi} \in \mathcal{L}\left(H_{+}^{2}(\Gamma)\right)$.

Proof. Let us consider the operators

$$
\begin{aligned}
& R_{1}:=\left(I-J_{\Gamma}\right) P_{\Gamma}: H_{+}^{2}(\Gamma) \rightarrow \operatorname{Im} Q_{J_{\Gamma}} \\
& R_{2}:=\frac{1}{2} P_{\Gamma}\left(I-J_{\Gamma}\right): \operatorname{Im} Q_{J_{\Gamma}} \rightarrow H_{+}^{2}(\Gamma) .
\end{aligned}
$$

These operators are inverses to one another and a direct computation yields that

$$
\overrightarrow{T_{\phi}} R_{1}=T_{\phi}-H_{\phi}
$$

which shows explicitly the equivalence relation between the operators $\vec{T}_{\phi}$ and $T_{\phi}-H_{\phi}$.

Proposition 5.3. Let $\phi \in \mathcal{G} L^{\infty}(\Gamma)$ and $\psi(t)=\phi^{-1}\left(-t^{-1}\right), t \in \Gamma$. The operator $\overrightarrow{T_{\phi}}: \operatorname{Im} Q_{J_{\Gamma}} \rightarrow H_{+}^{2}(\Gamma)$ is invertible if and only if $\overleftarrow{T_{\psi}}: H_{+}^{2}(\Gamma) \rightarrow \operatorname{Im} Q_{J_{\Gamma}}$ is invertible.

Proof. We will make use of Lemma 5.1 by choosing $P_{1}=Q_{J_{\Gamma}}, P_{2}=P_{\Gamma}, Q_{1}=P_{J_{\Gamma}}$ and $Q_{2}=Q_{\Gamma}$. Thus, from Lemma 5.1 we derive that $\overrightarrow{T_{\phi}}$ is invertible if and only if $P_{J_{\Gamma}} L\left(\phi^{-1}\right) Q_{\Gamma}$ is invertible. Multiplying from the left and the right in this last operator by $J_{\Gamma}$, we obtain

$$
J_{\Gamma} P_{J_{\Gamma}} L\left(\phi^{-1}\right) Q_{\Gamma} J_{\Gamma}=P_{J_{\Gamma}} J_{\Gamma} L\left(\phi^{-1}\right) J_{\Gamma} P_{\Gamma}
$$

Now, to reach the operator $\overleftarrow{T_{\psi}}$, we will consider the operator $V_{\Gamma}: L^{2}(\Gamma) \rightarrow L^{2}(\Gamma)$ $\left(V_{\Gamma} f\right)(t)=f(-t)$, and use it in (5.2) in the way that:

$$
V_{\Gamma} P_{J_{\Gamma}} J_{\Gamma} L\left(\phi^{-1}\right) J_{\Gamma} P_{\Gamma} V_{\Gamma}=Q_{J_{\Gamma}} \underbrace{V_{\Gamma} J_{\Gamma} L\left(\phi^{-1}\right) J_{\Gamma} V_{\Gamma}}_{L(\psi)} P_{\Gamma}=\overleftarrow{T_{\psi}}
$$

where $\psi(t)=\phi^{-1}\left(-t^{-1}\right)$.

We assemble in the next corollary a direct consequence of the last two propositions.

Corollary 5.4. Let $\phi \in \mathcal{G} L^{\infty}(\Gamma)$. Then the following assertions are equivalent:

(i) $T_{\phi}-H_{\phi}$ is invertible in $\mathcal{L}\left(H_{+}^{2}(\Gamma)\right)$,

(ii) $\vec{T}_{\phi}$ is invertible in $\mathcal{L}\left(\operatorname{Im} Q_{J_{\Gamma}}, H_{+}^{2}(\Gamma)\right)$,

(iii) $\overleftarrow{T_{\psi}}$ is invertible in $\mathcal{L}\left(H_{+}^{2}(\Gamma), \operatorname{Im} Q_{J_{\Gamma}}\right)$, where $\psi(t)=\phi^{-1}\left(-t^{-1}\right)$ 
Proposition 5.5. Assume that $\phi \in \mathcal{G} L^{\infty}(\Gamma)$ admits a weak odd asymmetric factorization in $L^{2}(\Gamma)$ with index $k=0$. Then the following assertions hold:

(i) the operator $\mathscr{E}=L\left(\phi_{o}^{-1}\right)\left(I+J_{\Gamma}\right) P_{\Gamma} L\left(\phi_{-}^{-1}\right)$ is a well-defined linear operator acting from $X_{1}$ into $X_{2}$,

(ii) $\overrightarrow{T_{\phi}} \mathscr{E}=P_{\Gamma \mid X_{1}}$,

(iii) $\operatorname{Ker} \vec{T}_{\phi}=\{0\}$.

Proof. (i) Let $f \in X_{1}$ and $\phi=\phi_{-} \phi_{o}$ (with $\phi_{-}$and $\phi_{o}$ under the conditions of Definition 4.1). We will compute $\mathscr{E} f$. First, we write $f(t)=\left(1-t^{-1}\right) f_{1}(t)$ with $f_{1} \in \mathfrak{R}$. Multiplying both sides of the last equality by $\phi_{-}^{-1}$, we have:

$$
\phi_{-}^{-1}(t) f(t)=\left(1-t^{-1}\right) \phi_{-}^{-1}(t) f_{1}(t) .
$$

Hence, we can decompose $\phi_{-}^{-1} f$ in an unique way:

$$
\phi_{-}^{-1}(t) f(t)=u_{1}(t)+p_{1}(t),
$$

where $u_{1} \in t^{-1} H_{-}^{2}(\Gamma)$ and $p_{1}$ is a polynomial. From the last equality and from the assumption that $f \in X_{1}$ it also follows that $f$ has the following form: $f(t)=\phi_{-}(t)\left(u_{1}(t)+p_{1}(t)\right)$. Later on we will use this fact. Now, applying the Riesz projection to $(5.3)$, we will have $P_{\Gamma}\left(\phi_{-}^{-1} f\right)(t)=p_{1}(t)$. Hence $(\mathscr{E} f)(t)=$ $\phi_{o}^{-1}(t)\left(p_{1}(t)+t^{-1} p_{1}\left(t^{-1}\right)\right)$. Since $p_{1}(t)+t^{-1} p_{1}\left(t^{-1}\right)$ vanishes at $t=-1$, this expression is $\left(1+t^{-1}\right)$ times a trigonometrical polynomial $f_{2}$, such that $f_{2}(t)=f_{2}\left(t^{-1}\right)$. Now it is clear that $\mathscr{E} f$ belongs to the space $X_{2}$.

(ii) Let us take again $f \in X_{1}$ and assume the existence of a weak odd asymmetric factorization of $\phi=\phi_{-} \phi_{o}$ in $L^{2}(\Gamma)$ (with index $k=0$ ). From the part $(i)$ of the proof we know that

$$
f(t)=\phi_{-}(t)\left(u_{1}(t)+p_{1}(t)\right),
$$

where $u_{1}$ and $p_{1}$ are as in the formula (5.3). Our aim is to compute $\overrightarrow{T_{\phi}} \mathscr{E} f$. We have already calculated $(\mathscr{E} f)(t)=\phi_{o}^{-1}(t)\left(p_{1}(t)+t^{-1} p_{1}\left(t^{-1}\right)\right)$. From here we have that

$$
\begin{aligned}
\left(\overrightarrow{T_{\phi}} \mathscr{E} f\right)(t) & =\left(P_{\Gamma} L\left(\phi_{-}\right) L\left(\phi_{o}\right) \frac{I-J_{\Gamma}}{2} \phi_{o}^{-1}\left(p_{1}+t^{-1} \widetilde{p_{1}}\right)\right)(t) \\
& =P_{\Gamma}\left(\phi_{-}\left(p_{1}+t^{-1} \widetilde{p_{1}}\right)\right)(t)
\end{aligned}
$$

In addition, we need to prove that $\overrightarrow{T_{\phi}} \mathscr{E}=P_{\Gamma \mid X_{1}}$. To this end, we need to show that the following inclusion holds true: $\phi_{-}\left(p_{1}+t^{-1} \widetilde{p_{1}}\right)-f \in t^{-1} H_{-}^{1}(\Gamma)$. We are left to note that the last inclusion was already deduced (even in a more general setting) in [2, Lemma 4.1]. This proves the part (ii) of the proposition.

(iii) For $f \in \operatorname{Ker} \overrightarrow{T_{\phi}}$, we directly have $f \in \operatorname{Im} Q_{J_{\Gamma}}$ and

$$
P_{\Gamma}(\phi f)=0 \text {. }
$$

Define $f_{-}:=\phi f$. From the definition of $P_{\Gamma}$ and (5.4) it follows that $f_{-} \in$ $t^{-1} H_{-}^{2}(\Gamma)$. Consequently, we have

$$
\phi_{-}^{-1} f_{-}=\phi_{o} f,
$$


and therefore

$$
t\left(1-t^{-1}\right) \phi_{-}^{-1}(t) f_{-}(t)=(t-1) \phi_{o}(t) f(t)=: \psi(t) .
$$

Additionally, we have that $\left(1-t^{-1}\right) \phi_{-}^{-1} \in H_{-}^{2}(\Gamma)$ and $t f_{-} \in H_{-}^{2}(\Gamma)$. Then it follows from (5.5) that $\psi \in H_{-}^{1}(\Gamma)$. Moreover, from the last identity in (5.5) we have $\widetilde{\psi}=-\psi$. In particular, this implies that $\psi=0$ and consequently $f=0$.

5.2. Proof of Theorem 3.4. After all the previous auxiliary material, we are now ready to present the proof of the invertibility criterion for $\mathrm{WH}_{\varphi}$.

Proof of Theorem 3.4. Let $\varphi \in \mathcal{G} L^{\infty}(\mathbb{R})$. First of all observe that

$$
W H_{\varphi}: L_{+}^{2}(\mathbb{R}) \rightarrow L^{2}\left(\mathbb{R}_{+}\right)
$$

is equivalent to $T_{\left(B_{0} \varphi\right)}-H_{\left(B_{0} \varphi\right)}: H_{+}^{2}(\Gamma) \rightarrow H_{+}^{2}(\Gamma)$. Indeed, if we consider the operator

$$
\mathcal{F}^{-1} B\left[T_{\left(B_{0} \varphi\right)}-H_{\left(B_{0} \varphi\right)}\right] B^{-1} \mathcal{F}: L_{+}^{2}(\mathbb{R}) \rightarrow L_{+}^{2}(\mathbb{R}),
$$

then a straightforward computation leads to

$$
\begin{aligned}
& \mathcal{F}^{-1} B\left[T_{\left(B_{0} \varphi\right)}-H_{\left(B_{0} \varphi\right)}\right] B^{-1} \mathcal{F}= \mathcal{F}^{-1} B\left[P_{\Gamma} L\left(B_{0} \varphi\right)\left(I-J_{\Gamma}\right) P_{\Gamma}\right] B^{-1} \mathcal{F} \\
&= \underbrace{\mathcal{F}^{-1} B P_{\Gamma} B^{-1} \mathcal{F}}_{\ell_{0} r_{+}} \mathcal{F}^{-1} \underbrace{B L\left(B_{0} \varphi\right) B^{-1}}_{L(\varphi)} \mathcal{F} \\
& \underbrace{\mathcal{F}^{-1} B\left(I-J_{\Gamma}\right) B^{-1} \mathcal{F}}_{I+J} \underbrace{\mathcal{F}^{-1} B P_{\Gamma} B^{-1} \mathcal{F}}_{\ell_{0} r_{+}} \\
&= \ell_{0} r_{+} \mathcal{F}^{-1} \varphi \mathcal{F}(I+J) \ell_{0} r_{+} \\
&: L_{+}^{2}(\mathbb{R}) \rightarrow L_{+}^{2}(\mathbb{R}) .
\end{aligned}
$$

We notice that in the last identities the formulas (4.4), (4.5) and (4.6) were used. In addition, it is clear that we can drop the last $\ell_{0} r_{+}$operator in (5.6) since this is just the identity operator in $L_{+}^{2}(\mathbb{R})$. So, we have that

$$
\mathcal{F}^{-1} B\left[T_{\left(B_{0} \varphi\right)}-H_{\left(B_{0} \varphi\right)}\right] B^{-1} \mathcal{F}=\ell_{0} W H_{\varphi} .
$$

This means that (as announced above) $W H_{\varphi}$ is equivalent to $T_{\left(B_{0} \varphi\right)}-H_{\left(B_{0} \varphi\right)}$ (due to the fact that $\ell_{0}: L^{2}\left(\mathbb{R}_{+}\right) \rightarrow L_{+}^{2}(\mathbb{R})$ is an invertible operator, the inverse being $\left.r_{+}: L_{+}^{2}(\mathbb{R}) \rightarrow L^{2}\left(\mathbb{R}_{+}\right)\right)$. Therefore, $W H_{\varphi}$ is invertible if and only if $T_{\left(B_{0} \varphi\right)}-H_{\left(B_{0} \varphi\right)}$ is invertible.

The next step is then to prove that the invertibility of $T_{\left(B_{0} \varphi\right)}-H_{\left(B_{0} \varphi\right)}$ happens if and only if $B_{0} \varphi$ admits an odd asymmetric factorization in $L^{2}(\Gamma)$ with index $k=0$.

If $T_{\left(B_{0} \varphi\right)}-H_{\left(B_{0} \varphi\right)}$ is invertible, then by Corollary 5.4 it follows that:

(a) $\overrightarrow{T_{\left(B_{0} \varphi\right)}}$ is invertible;

(b) $\overleftarrow{T_{\psi}}$ is also invertible (with $\psi(t)=\left(B_{0} \varphi\right)^{-1}\left(-t^{-1}\right)$ ) 
From (a), it follows that $\operatorname{Im} \overrightarrow{T_{\left(B_{0} \varphi\right)}}=H_{+}^{2}(\Gamma)$. Let us now consider $h_{o} \in \operatorname{Im} Q_{J_{\Gamma}}$ such that $\overrightarrow{T_{\left(B_{0} \varphi\right)}} h_{o}=1$. In addition, take $h_{-}(t):=\left(B_{0} \varphi\right)(t) h_{o}(t)$. From here we have that $h_{-} \in H_{-}^{2}(\Gamma)$ and $h_{-} \neq 0$. If defining

$$
\begin{aligned}
f_{-}(t) & :=\left(1+t^{-1}\right)^{-1} h_{-}(t), \\
f_{o}(t) & :=\left(1+t^{-1}\right)^{-1} h_{o}(t)
\end{aligned}
$$

it is readily seen that $|1+t| f_{o} \in L^{2}(\Gamma)$ and $f_{o}$ is an odd function. Indeed, for all $t \in \Gamma$, it holds true

$$
\begin{aligned}
f_{o}\left(t^{-1}\right) & =(1+t)^{-1} h_{o}\left(t^{-1}\right)=-(1+t)^{-1} t h_{o}(t)=-\left(1+t^{-1}\right)^{-1} h_{o}(t) \\
& =-f_{o}(t) .
\end{aligned}
$$

In addition, we obtain the "factorization"

$$
f_{-}=\left(B_{0} \varphi\right) f_{o}
$$

with

$$
\left(1+t^{-1}\right) f_{-} \in H_{-}^{2}(\Gamma), \quad|1+t| f_{o} \in L_{\text {odd }}^{2}(\Gamma) .
$$

From (b), it follows that the corresponding adjoint operator $\left(\overleftarrow{T_{\psi}}\right)^{*}=\vec{T}_{\bar{\psi}}$ is also invertible (recalling that $\left.\psi(t)=\left(B_{0} \varphi\right)^{-1}\left(-t^{-1}\right)\right)$. Thus, from the just presented reasoning, we guarantee the existence of elements $f_{-} \neq 0$ and $f_{o}$ such that

$$
\left(1+t^{-1}\right) f_{-} \in H_{-}^{2}(\Gamma), \quad|1+t| f_{o} \in L_{\text {odd }}^{2}(\Gamma),
$$

and

$$
f_{-}(t)=\overline{\left(B_{0} \varphi\right)^{-1}\left(-t^{-1}\right)} f_{o}(t) .
$$

Let us now pass to the complex conjugate and make the substitution $t \mapsto-t^{-1}$. Choosing $g_{-}(t)=\overline{f_{-}\left(-t^{-1}\right)}$ and $g_{o}(t)=\overline{f_{o}\left(-t^{-1}\right)}$, it follows that

$$
\left(1-t^{-1}\right) g_{-} \in H_{-}^{2}(\Gamma), \quad|1-t| g_{o} \in L_{\text {odd }}^{2}(\Gamma),
$$

and

$$
g_{-}(t)=\left(B_{0} \varphi\right)^{-1}(t) g_{o}(t) .
$$

Multiplying now the corresponding elements in the identities (5.7) and (5.8), we obtain $g_{-} f_{-}=g_{o} f_{o}$. Moreover, it follows that $g_{-} f_{-}=g_{o} f_{o}=: C$ is a nonzero constant (this can be verified in a similar way as in the proof of the uniqueness of weak odd asymmetric factorizations in $\left.L^{2}(\Gamma)\right)$.

Now, we put $\left(B_{0} \varphi\right)_{-}=f_{-}=C g_{-}^{-1}$ and $\left(B_{0} \varphi\right)_{o}=f_{o}^{-1}=g_{o} C^{-1}$. Hence,

$$
B_{0} \varphi=\left(B_{0} \varphi\right)_{-}\left(B_{0} \varphi\right)_{o},
$$

and we have shown that $B_{0} \varphi$ admits a weak odd asymmetric factorization in $L^{2}(\Gamma)$ (with index $k=0$ ). Now we have to prove that

$$
\mathscr{E}=L\left(\left(B_{0} \varphi\right)_{o}^{-1}\right)\left(I+J_{\Gamma}\right) P_{\Gamma} L\left(\left(B_{0} \varphi\right)_{-}^{-1}\right)
$$


can be extended to a linear bounded operator which acts on $L^{2}(\Gamma)$. From Proposition 5.5 we have that this $\mathscr{E}$ is well-defined. Assertion $(i i)$ of the same proposition gives the following:

$$
\mathscr{E}=\left(\bar{T}_{\left(B_{0} \varphi\right)}\right)^{-1} P_{\Gamma \mid X_{1}}
$$

(recall that $\overrightarrow{T_{\left(B_{0} \varphi\right)}}$ is invertible due to the hypothesis on $T_{\left(B_{0} \varphi\right)}-H_{\left(B_{0} \varphi\right)}$ ). Obviously, this right hand side can be extended by continuity to a linear bounded operator acting from $L^{2}(\Gamma)$ into $\operatorname{Im} Q_{J_{\Gamma}}$ (since that is a restriction of such an operator to the space $X_{1}$ ), and hence the present operator $\mathscr{E}$ can also be extended as well.

Let us now assume that $B_{0} \varphi$ admits an odd asymmetric factorization in $L^{2}(\Gamma)$ with index $k=0$ (and so the corresponding conditions $(i)-(i i i)$ of the Definition 4.3 are satisfied). By $\widetilde{\mathscr{E}}$ we will denote the continuous extension of the operator $\mathscr{E}=L\left(\left(B_{0} \varphi\right)_{o}^{-1}\right)\left(I+J_{\Gamma}\right) P_{\Gamma} L\left(\left(B_{0} \varphi\right)_{-}^{-1}\right)$. As far as $X_{1}$ is dense in $L^{2}(\Gamma)$, we have that

$$
\overrightarrow{T_{\left(B_{0} \varphi\right)}} \widetilde{\mathscr{E}}=P_{\Gamma}
$$

for operators defined in $L^{2}(\Gamma)$. In particular, this shows that $\widetilde{\mathscr{E}}_{\mid H_{+}^{2}(\Gamma)}$ is the right inverse of $\overrightarrow{T_{\left(B_{0} \varphi\right)}}$. Moreover, from the above identity we obtain

$$
\overrightarrow{T_{\left(B_{0} \varphi\right)} \widetilde{\mathscr{E}}_{\mid H_{+}^{2}(\Gamma)}} \overrightarrow{T_{\left(B_{0} \varphi\right)}}=\overrightarrow{T_{\left(B_{0} \varphi\right)}},
$$

and from here we have

$$
\overrightarrow{T_{\left(B_{0} \varphi\right)}}\left(\widetilde{\mathscr{E}}_{\mid H_{+}^{2}(\Gamma)} \overrightarrow{T_{\left(B_{0} \varphi\right)}}-I\right)=0 .
$$

Recalling now that the kernel of $\overrightarrow{T_{\left(B_{0} \varphi\right)}}$ is trivial (cf. Proposition $5.5(\mathrm{iii})$ ), it follows from (5.9) that $\widetilde{\mathscr{E}}_{\mid H_{+}^{2}(\Gamma)} \overrightarrow{T_{\left(B_{0} \varphi\right)}}=I$. Consequently, $\overrightarrow{T_{\left(B_{0} \varphi\right)}}$ is invertible and its inverse is just $\widetilde{\mathscr{E}}_{\mid H_{+}^{2}(\Gamma)}$. In such a case, observe that from Corollary 5.4 we conclude that $T_{\left(B_{0} \varphi\right)}-H_{\left(B_{0} \varphi\right)}$ is also an invertible operator.

Therefore, until now we have proved that $W H_{\varphi}$ is an invertible operator if and only if $B_{0} \varphi$ admits an odd asymmetric factorization in $L^{2}(\Gamma)$ with index $k=0$.

In addition, due to Proposition 4.4, we have that $B_{0} \varphi$ admits an odd asymmetric factorization in $L^{2}(\Gamma)$ with index $k=0$ if and only if $B_{0}^{-1}\left(B_{0} \varphi\right)=\varphi$ admits an odd asymmetric factorization in $L^{2}(\mathbb{R})$ with index $k=0$.

Putting altogether, we have that $W H_{\varphi}$ is invertible if and only if $\varphi$ admits an odd asymmetric factorization in $L^{2}(\mathbb{R})$ with index $k=0$.

\section{FREDHOLM PROPERTY}

In the present section it will be obtained a Fredholm criterion for $W H_{\varphi}$. Besides this, other particular results will follow as direct consequences of this Fredholm criterion. 
Theorem 6.1. Let $\varphi \in \mathcal{G} L^{\infty}(\mathbb{R})$. The operator $W H_{\varphi}: L_{+}^{2}(\mathbb{R}) \rightarrow L^{2}\left(\mathbb{R}_{+}\right)$is a Fredholm operator if and only if $\varphi$ admits an odd asymmetric factorization in $L^{2}(\mathbb{R})$. Moreover, if $\mathrm{WH}_{\varphi}$ is a Fredholm operator, then it holds

$$
\operatorname{dim} \operatorname{Ker} W H_{\varphi}=\max \{0,-k\}, \quad \operatorname{dim} \operatorname{Ker} W H_{\varphi}^{*}=\max \{0, k\},
$$

where $k$ is the index of an odd asymmetric factorization of $\varphi$ in $L^{2}(\mathbb{R})$.

Proof. Assume that $\mathrm{WH}_{\varphi}$ is a Fredholm operator with index $-k$. We will start by using the classical Wiener-Hopf technique to built a corresponding auxiliary invertible operator. For this purpose, let us consider the auxiliary function $\psi(x)=$ $\left(\frac{x-i}{x+i}\right)^{-k} \varphi(x)$. It is well-known that an Hankel operator with a continuous symbol is compact. Therefore (since for $k \in \mathbb{Z}$ the element $\zeta^{-k}$ with $\zeta(x):=\left(\frac{x-i}{x+i}\right)$ is continuous in the compactified real line), by employing formula (2.1), it follows that

$$
W H_{\psi}=W H_{\zeta^{-k}} \ell_{0} W H_{\varphi}+K_{1},
$$

where $K_{1}$ is a compact operator. In addition, let us also observe that $W H_{\zeta^{-k}}=$ $W_{\zeta^{-k}}+K_{2}$ (where $K_{2}$ is a compact operator). Thus, from the Fredholm theory of Wiener-Hopf operators we conclude that $W H_{\zeta^{-k}}$ is a Fredholm operator with Fredholm index $k$. Consequently, from identity (6.2) we conclude that $W_{\psi}$ is a Fredholm operator with index zero.

Let us now consider the Lebesgue measure zero set

$$
\mathbb{V}_{\psi}:=\{x \in \mathbb{R}: \psi(x)=\psi(-x)=0\}
$$

(note that $\psi \in \mathcal{G} L^{\infty}(\mathbb{R})$ ), and the corresponding characteristic function

$$
\chi_{\mathbb{V}_{\psi}}(x)= \begin{cases}1, & x \in \mathbb{V}_{\psi} \\ 0, & x \notin \mathbb{V}_{\psi}\end{cases}
$$

Arguing in a similar way as in the Toeplitz plus Hankel case (see [2]), it follows that

$$
\operatorname{Ker} W H_{\psi} \cong \operatorname{Im} W H_{\chi_{\mathbb{v}_{\psi}}} \text { or } \operatorname{Ker} W H_{\psi}^{*}=\{0\},
$$

where $\cong$ denotes the existence of an isomorphically isomorphism between the related sets. Since $\mathbb{V}_{\psi}$ has zero Lebesgue measure, and hence $\chi_{\mathbb{V}_{\psi}}=0$ for almost all $x \in \mathbb{R}$, it follows that $\operatorname{Im} W H_{\chi_{\mathbb{V} \varphi}}=\{0\}$. This combined with (6.3) leads to

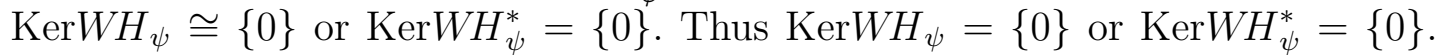
This means that $W H_{\psi}$ is invertible (since we have already previously concluded that $W H_{\psi}$ is a Fredholm operator with index zero).

Now, employing Theorem 3.4 we deduce that $\psi$ admits an odd asymmetric factorization in $L^{2}(\mathbb{R})$ with index zero. Hence $\varphi$ admits an odd asymmetric factorization in $L^{2}(\mathbb{R})$ with index $k$.

Now we will proceed with the reverse implication. Assume that $\varphi$ admits an odd asymmetric factorization in $L^{2}(\mathbb{R})$ with index $k$. Consequently, we have a corresponding operator decomposition:

$$
W H_{\varphi}=W_{\varphi_{-}} \ell_{0} W H_{\zeta^{k}} \ell_{0} W H_{\varphi_{o}}+K,
$$


where $K$ is a compact operator. Thus, $W H_{\varphi}$ is a Fredholm operator if and only if $W_{\varphi_{-}} \ell_{0} W H_{\zeta^{k}} \ell_{0} W H_{\varphi_{o}}$ is a Fredholm operator. However, the latter operator is equivalent to $W H_{\zeta^{k}}$, because $\ell_{0}, W_{\varphi_{-}}$and $W H_{\varphi_{o}}$ are invertible operators. Therefore, as above, we simply have to notice that $W H_{\zeta^{k}}=W_{\zeta^{k}}+H_{\zeta^{k}}$ is a Fredholm operator with index $-k$.

Let us now turn to formulas (6.1). Under the Fredholm property we already know that $W_{\varphi}$ has a Fredholm index equal to $-k$. Thus, combining this fact with (6.3) it directly follows the presented formulas for the defect numbers.

As a direct consequence of the last result we collect the following interesting conclusions.

Corollary 6.2. If $\mathrm{WH}_{\varphi}$ is a Fredholm operator, then $\mathrm{WH}_{\varphi}$ has a trivial kernel or a trivial cokernel.

Corollary 6.3. The Wiener-Hopf plus Hankel operator $\mathrm{WH}_{\varphi}$ is invertible if and only if $\mathrm{WH}_{\varphi}$ is Fredholm with index zero.

In addition, it is also clear that Theorem 6.1 implies Theorem 3.4 but we would like to emphasize that to prove Theorem 6.1 we needed to use Theorem 3.4.

Acknowledgement: This work was supported in part by Unidade de Investigação Matemática e Aplicações of Universidade de Aveiro, Portugal, and the Portuguese Science Foundation (FCT-Fundação para a Ciência e a Tecnologia).

\section{REFERENCES}

1. E.L. Basor and T. Ehrhardt, On a class of Toeplitz + Hankel operators, New York J. Math., 5 (1999), 1-16.

2. E.L. Basor and T. Ehrhardt, Factorization theory for a class of Toeplitz + Hankel operators, J. Oper. Theory, 51 (2004), 411-433.

3. G. Bogveradze and L.P. Castro, Wiener-Hopf plus Hankel operators on the real line with unitary and sectorial symbols, Contemp. Math., 414 (2006), 77-85.

4. G. Bogveradze and L.P. Castro, On the Fredholm index of matrix Wiener-Hopf plus/minus Hankel operators with semi-almost periodic symbols, Operator Theory: Advances and Applications, 181 (2008), 143-158.

5. G. Bogveradze and L.P. Castro, On the Fredholm property and index of Wiener-Hopf plus/minus Hankel operators with piecewise almost periodic symbols, Appl. Math. Inform. Mech., to appear.

6. A. Böttcher, Yu.I. Karlovich and I.M. Spitkovsky, Convolution Operators and Factorization of Almost Periodic Matrix Functions, Birkhäuser, Basel, 2002.

7. L.P. Castro, R. Duduchava and F.-O. Speck, Asymmetric factorizations of matrix functions on the real line, Operator Theory: Advances and Applications, 170 (2006), 53-74.

8. L.P. Castro, F.-O. Speck and F. S. Teixeira, A direct approach to convolution type operators with symmetry, Math. Nachr., 269-270 (2004), 73-85.

9. L.P. Castro, F.-O. Speck and F. S. Teixeira, Mixed boundary value problems for the Helmholtz equation in a quadrant, Integral Equations Oper. Theory, 56 (2006), 1-44.

10. V.G. Kravchenko, A.B. Lebre and J.S. Rodríguez, Factorization of singular integral operators with a Carlemen shift via factorization of matrix functions: The anticommutative case, Math. Nachr., 280 (2007), 1157-1175.

11. A.P. Nolasco, Regularity Properties of Wiener-Hopf-Hankel Operators, Ph.D. Thesis, University of Aveiro, 2007. 
12. A.P. Nolasco and L.P. Castro, Factorization theory for Wiener-Hopf plus Hankel operators with almost periodic symbols, Contemp. Math., 414 (2006), 111-128.

13. A.P. Nolasco and L.P. Castro, A Duduchava-Saginashvili's type theory for Wiener-Hopf plus Hankel operators, J. Math. Anal. Appl., 331 (2007), 329-341.

14. F.-O. Speck: General Wiener-Hopf Factorization Methods, Research Notes in Mathematics, 119, Pitman, London, 1985.

1,2 Research Unit "Mathematics and Applications", Department of Mathematics, University of Aveiro, 3810-193 Aveiro, Portugal.

E-mail address: giorgi@ua.pt, castro@ua.pt 